\title{
Expert Review: Current Trends of Journal of Health and Medical Informatics
}

\section{Diana Y Zhao*}

Executive Deputy Dean Sino-Dutch Biomedical and Information Engineering School of Northeastern University, Shenyang, China

Medical Informatics was first used in basic research, clinical practice and hospital management as a MeSH term in 1987, and is now extended to the regional health information system and the National Health Information Network. It is the field of information science concerned with the analysis and dissemination of medical data through the application of computers to various aspects of health care and medicine.

Bibliometric studies carried out by scholars in top journals in this area in recent years show that high-frequency terminologies in the field of health and medical informatics focused on two major categories and six small classes. Of which, the online community and professional healthcare websites in the first category are gradually gaining popularity among patients and health workers. Researchers should pay more attention to people's online searching behaviors for public health information and its impact on the improvement of health habits. In the classification of health information systems construction and application, a hospital information system covers a wide scope. There are systems for hospital management including the outpatient information systems and clinical management systems, and also systems for diagnosis and treatment such as the medical imaging systems and the automatic medical advice entry systems. As for the Decision Support System, performance appraisal systems support administrative decisions, while the expert diagnostic system is geared to the needs of health workers and patients. With regard to the storage and handling systems for patients' information, thorough researchers in Europe and America carried out in-depth research on Electronic Medical Records (EMR) and Electronic Health Records (EHR) since the 1990s, there is still considerable potential for further research in this area, such as the construction of community and regional health information platform based on EHR, and text data mining of EMR base on natural language processing. Meanwhile, with the implementation of the health information system on a large scale, its effect evaluation began to receive attention of researchers.

In the other category, the first and foremost is the applications and research of algorithms in biomedicine. In the medical and healthcare field, issues similar to whether a new treatment method is superior to the conventional ones are always emerging. Analysis of assessment to solve such problems, of which cost-effectiveness analysis is most common. Such kind of online self-testing system may not only improve the security level, but will also increase patients' satisfaction with nursing, and thus it is suitable for construction and popularization.

We are aware that the discovery process for the laws of biomedicine tends to observe certain phenomenon first, and then validate it by using statistical models to determine the relationship between the variables. Relationship analysis and application is therefore widespread. Variables certainly have their place. In addition to the study of patients' behavior, that of the doctors also needs to be included. It indicates that the change of medical models forces more and more researchers to begin to pay close attention to the relationship between social behavior and disease.

Along with the large-scale applications of information technology such as internet and database in medical treatment, medical consultation, prevention and healthcare, health management, and medical education, etc., the variety of health information systems have started to spread. The decision support systems, performance appraisal systems, and expert diagnosis systems have been widely discussed. Accordingly, the construction and implementation of these systems, program development, output assessment, and cost-benefit analysis have become research hotspots. Other than the information technologies and their applications, health information methods study is also a major research direction. RCT, Meta-analysis, evidencebased medicine, the EM algorithms, regression model and a series of statistical models and methods have been applied to areas such as the disease risk management, therapeutic efficacy assessment, and health behavior change, etc. Meanwhile, issues including the ways to reduce statistical bias, and the determination of the appropriate sample size depending on aims are also taken seriously by researchers. Moreover, general medicine, primary healthcare and management of stroke have become the new directions for health management.

We may say that research hotspots for medical informatics in recent years have been concentrated in two dimensions, namely the application of information technology and the health information analysis methods. In terms of the application dimension, researchers have paid much attention to the change of people's health behavior caused by modern information technology

and thus have conducted a great deal of work in the field. Health information systems with various features are widely promoted. In the meantime, the performances of evaluation, testing and costbenefit analysis have become the focus of research. With regard to the method dimension, algorithm and model-based analytical methods are continually being enhanced to adapt to the growing amount of complex health information data. The bioinformatics algorithms, analytical methods in assessment and analytical methods in relationship are the three major research directions in health information analysis. The study found that the two dimensions are not isolated from each other, but promote mutual cross integration. On the one hand, technology applications facilitated the follow-up by research methods. Such as with the large-scale application of health information systems, the corresponding assessment methods appeared in large numbers. On the other hand, new approaches provided theoretical guarantee for the development and application of technology. A good example is the emergence of artificial neural network algorithm has enhanced

${ }^{*}$ Corresponding author: Diana Y. Zhao, Executive Deputy Dean Sino-Dutch Biomedical and Information Engineering School of Northeastern University, Shenyang, China, Tel: +86-24-83676665; E-mail: zhaoyue@bmie.neu.edu.cn

Received July 14, 2014; Accepted July 15, 2014; Published August 04, 2014

Citation: Zhao DY (2014) Expert Review: Current Trends of Journal of Health and Medical Informatics. J Health Med Informat. 5: e130. doi:10.4172/2157$7420.1000 \mathrm{e} 130$

Copyright: () 2014 Zhao DY. This is an open-access article distributed under the terms of the Creative Commons Attribution License, which permits unrestricted use, distribution, and reproduction in any medium, provided the original author and source are credited. 
the availability of clinical decision support systems. Obviously, the applications extending and methods innovation will become one of the future application directions.

It is not difficult to predict that the future application directions for health and medical informatics will more than likely incline to the implementation of new features, such as the development of health decision support system at strategic level, or more intelligent healthcare service networks, etc., and their clients tend more to be the general public. The current development trend of the global medical informatics is driven by such mission to be further overlapping and integrating with health care, health management and health policy.

Obliviously, with the above trends development, there must be a steadily growing presence and increasing visibility of health and medical informatics academic literature over the years. According to the relevant research, the average annual article publication growth rate was $12 \%$. Research output suggest it will be a maturing discipline, and highlight specific journals in which the health and medical informatics literature appears most frequently, especially on the above research trends. 